\title{
Self-Administered Optimism Training: Mechanisms of Change in a Minimally Supervised Psychoeducational Intervention
}

\author{
David M. Fresco \\ Michael T. Moore \\ Lisa Walt \\ Kent State University, Ohio \\ Linda W. Craighead \\ Emory University, Atlanta, Georgia
}

\begin{abstract}
Pessimistic explanatory style is a robust predictor of future depressive symptoms. There were 112 college students with a pessimistic explanatory style identified from a larger screening sample. Participants were randomly assigned to Self-Administered Optimism Training or a no-treatment control group. Participants assigned to SOT received 10 minutes of instruction regarding self-monitoring of how they assigned causes to events in their lives and "brainstorming" alternate causes, and then sent off to record daily diaries that captured this information every day for 28 days. The SOT participants demonstrated significant drops in pessimism in three separate but related assessments. Further, findings indicated a significant correlation between cognitive change and depression symptom change for the SOT group but not the control group. Although preliminary in nature, findings from the current study demonstrate the feasibility and preliminary efficacy of an inexpensive, prophylactic treatment for depression that utilizes a minimum of therapist contact.
\end{abstract}

Keywords: cognitive therapy; explanatory style; prevention

ognitive diathesis-stress theories of depression (Abramson, Seligman, \& Teasdale, 1978; Beck, 1967) have advanced our understanding of the etiology, maintenance, and treatment of depression. These theories posit that individuals who adopt a depressogenic view of the world are vulnerable to depression when confronted with negative life events. Specifically, vulnerability for depression in Beck's (1967) theory is associated with dysfunctional attitudes and negative schema regarding the self, world, and future. Similarly, the reformulated learned helplessness theory (Abramson et al., 1978) and hopelessness theory (Abramson, Metalsky, \& Alloy, 1989) conceptualize risk for depression in terms of a depressogenic or pessimistic explanatory style (the tendency to view negative events as arising from stable, global, and internal causes). The 
current investigation sought to evaluate the efficacy of an intervention designed to reduce pessimistic explanatory style and thereby reduce a hypothesized vulnerability for depression. Given that this intervention involved little actual therapist contact (only 10 minutes of instruction), we also sought to determine the efficacy of an intervention that is largely independent of nonspecific treatment effects such as the therapeutic alliance and empathy. Prior to a detailed discussion of the intervention itself, it is first necessary to understand the mechanism of risk for depression that it attempts to interrupt, the various theoretical permutations of the depressogenic cognitive style.

\section{Beck's Theory and Cognitive Therapy}

Beck's $(1967,1987)$ theory posits that depressed affect is heavily influenced by recurrent automatic thoughts with negative content. These thoughts arise from deeply held dysfunctional beliefs or schemas. When these schemas have content relating to the self, world, and future they are referred to as a negative cognitive triad, such as "If I ever fail, no one will love me," and these schemas become activated when a negative life event, such as failing an important test, primes these beliefs. Beck identified that schemas and automatic thoughts, and the depressed affect that results from them, tend to be self-perpetuating as the depressed person both attends more to negative events in their lives and interprets events that occur after the onset of the depressed mood negatively in light of their own dysfunctional cognitions.

Beck's cognitive therapy of depression (CT; Beck, Rush, Shaw, \& Emery, 1979) is one of the most empirically validated and efficacious treatments (Blackburn \& Moorhead, 2001; DeRubeis \& Crits-Cristoph, 1998). One of the primary mechanisms by which CT is proposed to effect change is by teaching depressed individuals to analytically monitor their own negative thoughts, independently of their current mood state, in such a way that they can identify and challenge the source of these thoughts. In so doing, the desired goal is that the depressed individual's thoughts can be altered to become less influenced by preexisting schemata, and that this will lead to a reduction in the symptoms of depression. In CT, this modification in thought patterns is initially undertaken with the therapist assisting in directly challenging the validity of the depressed client's thoughts in session, and eventually through the use of homework assignments, such as the Daily Record of Dysfunctional Thoughts (Beck et al., 1979, pp. 286-287), performed outside of therapy. These assignments involve the therapist teaching the client to independently monitor the content of their thoughts and to change this content by more effortful processing of events in their lives. It is thought that by being conducted outside of therapy, homework assignments also assist the client by more readily mimicking life outside of a therapeutic context, thereby facilitating generalization.

\section{Learned Helplessness Theory}

The reformulated learned helplessness theory (Abramson et al., 1978) views vulnerability to depression based on the way that individuals assign causes to the negative events that occur in their lives. The central concept in this theory is "explanatory style," which is defined as an individual's relatively stable tendencies in assigning causes to negative events. The reformulated learned helplessness theory is a cognitive diathesis-stress theory that posits stressful life events are more likely to result in depression when they are interpreted in light of a pessimistic explanatory style. Individuals are more likely to develop depression when stressful life events are accompanied by a tendency to view the cause of that event as due to internal ("It's all my fault"), stable ("The cause will exist to effect my life forever"), and global ("The cause influences all aspects of my life") causes. A pessimistic explanatory style is defined as the tendency to attribute negative events to internal, stable, and global causes, and positive events to external, unstable, and specific causes. Substantial research supports the diathesis-stress component of the reformulated learned helplessness theory. Specifically, the interaction of negative life events and a pessimistic explanatory style have been shown to reliably predict the onset of symptoms of depression in 
both psychiatric and undergraduate populations (Abela \& Seligman, 2000; Alloy \& Clements, 1998; Alloy et al., 1999; Metalsky, Joiner, Hardin, \& Abramson, 1993; Sweeney, Anderson, \& Bailey, 1986).

\section{Self-Administered Optimism Training}

The current investigation sought to evaluate the feasibility of an intervention, informed by both Beck's theory $(1967,1987)$ as well as the reformulated learned helplessness theory (Abramson et al., 1978), and that was designed to reduce current levels of pessimistic explanatory style. Selfadministered optimism training (SOT; Fresco \& Craighead, 1993) was developed as a minimally supervised analogue of cognitive behavior therapy (CBT) by teaching participants to self-monitor daily positive and negative events in much the way a patient enrolled in CBT would record with the daily record of dysfunctional thoughts (DRDT). Unlike the CBT as proposed by Beck and colleagues (1979), SOT incorporates theory from the reformulated learned helplessness theory by specifically assessing the attributions that participants assign to daily events. The SOT participants were asked to list the best and worst events of the day for 28 days, the initial cause that they attributed the events to, and then rate that cause on the attributional dimensions of internality, stability, and globality. After some reflection or brainstorming, participants were instructed to provide a revised cause for the daily best and worst events, and then to rerate the cause along attributional dimensions. The rationale for this intervention was that more effortful processing would result in less pessimistic attributions, which would then reduce symptoms of depression. However, it should be noted that SOT participants were not instructed in any way how to go about this process of identifying additional causes or what the expected outcome of this process would be. These instructions were administered in approximately 10 minutes in a group format by a graduate research assistant. Control group participants were administered baseline measures of attributional style, symptoms of depression, and other measures unrelated to the current investigation and scheduled a time to return 28 days later for their Time 2 assessment.

Thus, SOT represents a psychoeducational, attributional retraining intervention that focuses an individual's attention to daily events and the causes of these events via a daily diary. The individuals are taught to refocus their attention on additional or alternative causes and make new, more adaptive attributions similar to the methods of traditional CBT. One important difference between SOT and CBT is notable. Although SOT is directed at having participants identify additional causes, the CBT therapist also tries to foster the identification of more accurate or realistic cognitions. Given the minimal ongoing therapeutic input in SOT and the difficulty in assessing this aspect of attributional style (Alloy \& Abramson, 1988; Moore \& Fresco, 2007), explicitly attempting to mimic this aspect of CBT treatment was not feasible. A secondary goal of SOT was to begin the process of developing a protocol that was relatively inexpensive to administer and that might ultimately hold promise for the prevention of emotional problems related to a pessimistic explanatory style. In addition, the use of a minimally supervised format allowed for the evaluation of an intervention free from the common or nonspecific mechanisms of therapy.

\section{Active Ingredients in Psychotherapy}

Psychotherapy is as varied as the multitude of people and professions who practice it. Snyder, Michael, and Cheavens (1999) estimate that there are currently over 500 conceptually distinct therapeutic approaches. Despite this theoretical heterogeneity, numerous, well-designed studies have found little difference in the efficacy of these varied psychotherapy protocols (Luborsky, Singer, \& Luborsky, 1975; Smith, Glass, \& Miller, 1980; Wampold et al., 1997). This finding has led to the conclusion that the more common forms of psychological treatment are all effective to an equal degree when generalized across psychiatric populations and treatment settings. Rosenzweig (1936, p. 412) illustrated this result in his now well-known Dodo Bird's verdict-an allusion to 
the Dodo Bird in Alice in Wonderland who proclaimed that "Everyone has won and all must have prizes." One reason that is often given for this equivalence of psychosocial treatment efficacy is that psychotherapy, in its many theoretical guises, operates via mechanisms that are common to most therapeutic modalities (i.e., working alliance: Horvath \& Greenberg, 1994; empathy: Lafferty, Beutler, \& Crago, 1991) as opposed to factors unique to the particular intervention.

The use of a minimally supervised format also allowed for an evaluation of the efficacy of SOT independent of many of the aforementioned common or nonspecific mechanisms of therapy. While controlling for all of these nonspecific mechanisms is impractical in a single investigation, the current study represents an attempt to illustrate that an intervention utilizing primarily active ingredients can be efficacious. The SOT also represents an attempt to develop a treatment that can reduce the risk for depression and be administered inexpensively. Insofar as SOT is administered quickly, in a group format, and requires minimal training to administer, such a treatment would be much less costly than traditional individual psychotherapy. The treatment could therefore be evaluated for its ability to prevent depression in individuals without access to traditional psychotherapy due to its cost (i.e., the uninsured) or the lack of qualified professionals (i.e., in more rural communities).

Taken together, the current investigation sought to evaluate the feasibility of a prophylactic intervention, designed to reduce risk for depression, which can also be administered quickly, easily, and cheaply to large groups of at-risk individuals. As such, this project falls squarely into what Rounsaville, Carroll, and Onken (2001) define as stage I behavioral therapy research. This stage of research is focused on feasibility (manual development, psychometric evaluation, and pilot testing) and can be contrasted with the stage II focus on efficacy and the use of the randomized clinical trial. Self-administered optimism training is also thought to be relevant to the discussion regarding how therapy works in its ability to induce clinically significant change without the benefit of many (but not all) nonspecific therapeutic factors.

\section{Hypotheses}

1. Pessimistic college students who complete SOT will endorse lower levels of pessimistic explanatory style as compared to pessimistic college students in a no-treatment control group.

2. For the SOT group, decreases in pessimistic explanatory style will be associated with corresponding reductions in symptoms of depression. Insofar as minimal cognitive change is predicted for the control group, there is no specific prediction regarding the relationship between cognitive change and mood change in this group.

3. The SOT participants will experience reductions in pessimistic explanatory style over time as indexed with the ratings made in their daily diaries.

\section{METHOD}

\section{Procedures}

All data were obtained from students enrolled in introduction to psychology classes from a large, southeastern university. In return for their effort, all participants received course credit. Participants were screened with the Expanded Attributional Style Questionnaire (EASQ; Peterson \& Villanova, 1988) and asked to participate if their composite negative (CN; the average responses of internal, stable, and global items) score was in the upper quartile of the sample's distribution (the participants that compose the current sample represent slightly more than $25 \%$ of the total screening sample as a result of several individuals whose scores were right on the quartile cut off). The mean $\mathrm{CN}$ score for the screening sample $(n=386)$ was $4.46(S D=.46)$, while $\mathrm{CN}$ scores 
ranged from 2.65 to 5.76. The upper quartile of $\mathrm{CN}$ scores ranged from a score of 4.75 to 5.76 . The Beck Depression Inventory (BDI; Beck et al., 1979) was also completed at this screening, but only the EASQ was utilized in selecting eligible participants. Consenting participants were then randomly assigned to either the SOT or a no-treatment control group. Participants in the SOT group reported to small group training sessions, which consisted of approximately 10 minutes of instruction in: (a) self-monitoring daily negative and positive events; (b) identifying the initial cause, and rating that cause along the dimensions of internality stability and globality; (c) brainstorming additional or alternate causes; and (d) arriving at a revised cause that was also rated along the dimensions of internality, stability, and globality. To reduce the likelihood that demand characteristics produced the results obtained, participants were merely instructed to find alternate causes. No mention was ever made of how this process of arriving at alternate causes should be undertaken. After receiving this instruction, participants were sent home with a 28 -day supply of paper and pencil materials and told to complete journal entries over the course of the next month. Participants were asked to identify at least 5 positive and 5 negative events per week (for a total of 10) and to identify and rate the initial and revised causes of these events; but participants were free to complete more as needed. The SOT participants returned 7 day's worth of journal entries at the end of each week. In the intervening month, control participants were not involved in any tasks related to this study. Finally, both SOT and control participants returned shortly after the end of the month long SOT cycle to complete the Time 2 packet of self-report measures.

\section{Participants}

Of the 55 SOT participants, $12(22 \%)$ dropped out during the course of the study compared with $2(4 \%)$ of the original 57 control participants. This number is comparable to the $10 \%-50 \%$ attrition rates reported in other studies of cognitive therapy (Dobson et al., 2008; Oei \& Kazmierczak, 1997; Simons, Levine, Lustman, \& Murphy, 1984). A $\chi^{2}$ analysis indicated that this difference in attrition rates was statistically significant $\left(\chi^{2}(112)=63.00, p<.001\right)$. However, a number of demographic and study variables (age, sex, ethnicity, number of brothers or sisters, birth order, parents' marital status, the presence of a history of parental death, remarriage, self-reported history of major depressive disorder or dysthymic disorder, total BDI score, and ASQ-CN) did not differentiate participants who prematurely dropped out of treatment (attritors) from nonattritors in the current sample. Therefore, it is unlikely that differential attrition, while present, exerted a significant effect on the results obtained. In the final sample, $78 \%$ of all participants (both SOT and control group) were female and $22 \%$ were male and the mean age was 19.23 years of age (SD $=2.8$ years). Although most of our participants were female, this number is representative both of the screening sample from which they were sampled and the individuals at highest risk to develop depression (American Psychiatric Association, 2000). Approximately $80 \%$ of the total sample was White, $6 \%$ Black, $2 \%$ Asian, $1 \%$ Indian, $2 \%$ Arabic, and $9 \%$ had missing ethnicity data.

\section{Measures}

The EASQ (Peterson \& Villanova, 1988) is an expanded version of the Attributional Style Questionnaire (ASQ; Peterson et al., 1982; Seligman, Abramson, Semmel, \& von Baeyer, 1979). Both are self-report inventories that assess causal attributions for hypothetical negative events along the dimensions of internality, stability, and globality that are rated on a 1 to 7 scale. While the original ASQ consists of six positive and six negative hypothetical events, the EASQ consists of 24 negative hypothetical events and no hypothetical positive events. Higher ratings represent responses endorsing internal, stable, and global causes while lower ratings represent external, unstable, and specific causes. Consistent with the reformulated learned helplessness theory of depression (Abramson et al., 1978), a composite negative (CN) score is computed by averaging the values of 24 internality, stability, and globality items for the negative event statements. 
A composite positive (CP) score can also be computed by averaging the values of the 24 internality, stability, and globality items for the positive event statements.

The EASQ-CN scores demonstrate adequate internal consistency $(\alpha=.66-.88$; Peterson \& Villanova, 1988) and convergent validity $(r=0.18-0.40$ with a measure of symptoms of depression; Peterson \& Villanova, 1988). Small to moderately sized correlations (cf., Cohen, 1988) were found between the negative event dimensions on the EASQ and ratings made from actual, not hypothetical events ( $r=0.18-0.36$; Peterson \& Villanova, 1988). It should be noted that while both actual and hypothetical (from the EASQ) were rated on the same dimensions in the aforementioned Peterson and Villanova (1988) study, the content of these events differed from one another. The results from Peterson and Villanova (1988) are comparable to the $\alpha=.67$ for EASQ-CN at Time 1 and $\alpha=.74$ for EASQ-CN at Time 2 that was found in the current study. In addition, the original ASQ has been shown to have adequate test-retest reliability for the negative event dimensions ( $r=0.52-0.60$; Colin, Sweeney, \& Schaeffer, 1981; Peterson et al., 1982; Sweeney et al., 1986) in both psychiatric and undergraduate populations.

The EASQ-CP scores have demonstrated a much less consistent relationship to symptoms of depression than CN scores (Peterson, 1991; Robins, 1988; Sweeny et al., 1986) and the reformulated learned helplessness theory does not make explicit predictions concerning positive events (cf. Peterson \& Seligman, 1984). It is for these two reasons, and the lack of significant change in EASQ-CP scores over the course of $\mathrm{SOT}^{\mathrm{i}}$ that the positive events dimensions were not examined further in the current investigation.

The BDI (Beck et al., 1979) is a 21-item, self-report instrument that broadly assesses the symptoms of depression including the affective, cognitive, behavioral, somatic, and motivational components as well as suicidal wishes. The BDI demonstrates adequate internal consistency $(\alpha=.73-.92)$ and test-retest reliability $(0.48-0.86)$ in both psychiatric and student samples (Beck, Steer, \& Garbin, 1988). In addition, the BDI demonstrates good convergent validity with structured clinical interviews (Stukenberg, Dora, \& Kiecolt-Glaser, 1990) and a variety of other self-report scales of depression (Beck et al., 1988). Strong internal consistencies were found in the current study at Time $1(\alpha=.85)$ and Time $2(\alpha=.88)$.

The DAQ, which was created for this study, uses a format similar to the ASQ and was designed to record the best and worst actual (as opposed to hypothetical) events of the day, as well as their causes of those events, and ratings for the causes on dimensions of internality, stability, and globality. For the purposes of this investigation, space was also provided for participants to record revised causes of the day's best and worst events as a result of further reflection on them that are additionally rated on internality, stability, and globality. However, for the reasons cited above, investigation of attributions for the positive event dimensions was not undertaken. Internal consistency for the DAQ-CN items for initial $(\alpha=.95)$ and brainstormed ratings $(\alpha=.96)$ were excellent.

In addition to the self-reported scores on the DAQ, participants' responses were also evaluated using the content analysis of verbatim explanations (CAVE; Peterson, Schulman, Castellon, \& Seligman, 1992) procedure. Samples of verbatim speech or writing can be submitted to analysis with the CAVE technique wherein statements describing an event and its cause are extracted and then independently rated by trained coders on the dimensions of internality, stability, and globality (similar to the EASQ). For the current study, the events that participants recorded on the DAQ were analyzed using this technique. However, as mentioned above, investigation of attributions for the positive event dimensions was not undertaken. The CAVE raters were trained by having them read Peterson's CAVE scoring manual (Peterson et al., 1992) and practice-rating 100 hypothetical event-attribution statements. Interrater agreement was also calculated for three raters from a random sample of 750 extractions, which was taken from a pool of over 6,000 extractions collected as part of the aforementioned daily diary study. The extractions used in the current study were drawn from this pool and rated by the same three raters. An acceptable degree of interrater agreement was obtained $(\alpha=.82$, intraclass correlation $=.82)$, as predicted. Trained 
CAVE coders were kept blind to the identity of the participant, what day in the 28-day cycle the extraction was taken, and whether the extraction was from the initial or brainstormed trial.

The CAVE technique has been shown to have adequate to good internal consistency (for internality, stability, globality, and the composite negative scores $\alpha=.88, .62, .59$, and .85 , respectively; Abela, Fresco, Kostelnik, \& Payne, 2003), as well as test-retest reliability (for internality, stability, and globality $r=0.63-0.90$; Peterson, Bettes, \& Seligman, 1985; Schulman, Castellon, \& Seligman, 1989). The CAVE technique also demonstrates adequate concurrent validity as it was found to correlate $r=0.48$ and 0.52 for the composite negative and composite positive scores, respectively (Abela et al., 2003; Peterson et al., 1985; Schulman et al., 1989). In addition, it was found to correlate between $r=0.23$ to 0.36 with the BDI (Abela et al., 2003; Schulman et al., 1989). The CAVE ratings were found to have significantly higher agreement than chance with a structured diagnostic interview and to be able to discriminate between participants with generalized anxiety disorder and depression (Riskind, Castellon, \& Beck, 1989), illustrating both criterion and discriminate validity, respectively. Adequate to strong internal consistencies were found in the current study for internality $(\alpha=.69)$, stability $(\alpha=.91)$, globality $(\alpha=.95)$, and the CAVE-CN scale $(\alpha=.96)$.

\section{RESULTS}

There was a low percentage of missing EASQ data at both Time $1(M=.92 \%, S D=2.46 \%)$ and Time 2 (excluding treatment noncompleters; $M=1.41 \%, S D=4.27 \%$ ). To address the question of the relationship between missing data at Time 1 and attrition at Time 2, SOT completers and noncompleters were compared on the percent of EASQ data missing at Time 1 . The results of this analysis indicate that Time 1 missing data was equivalent between these two groups $(F[1,110]=.002$, $p=.96$, Cohen's $f=.00)$. The amount of missing DAQ data was more substantial $(M=33.95 \%$, $S D=26.39 \%)$. However, compliance rates found in research examining the use of similar homework assignments in CBT are uniformly below 50\% (48\%: Clarke et al., 1992; 39\%: Helbig \& Fehm, 2004; 50\%: Primakoff, Epstein, \& Covi, 1986). We dealt with missing data by utilizing an expectation-maximization (EM) imputation algorithm. ${ }^{2}$ The EM imputation has been found to yield better estimates of missing data points than many other commonly used procedures (e.g., mean imputation, regression imputation; Liang \& Bentler, 2004).

\section{Pretreatment Group Differences}

Chi-squared tests revealed that at pretreatment, no differences were found between participants in the treatment or control groups on sex, ethnicity, birth order, parents' marital status, the presence of a history of parental death, remarriage, or of self-reported history of major depressive disorder or dysthymic disorder in the participants. In addition, one-way ANOVA revealed no differences between the groups in age, number of brothers or sisters, total BDI score, or EASQ-CN.

\section{Change in Explanatory Style}

Repeated-measures ANOVA using EASQ-CN at Time 1 and Time 2 as the dependent measures and Group (2; SOT vs. Control) as a between subjects factor revealed a significant main effect for Time, $F(1,110)=43.94, p<.001$ (Cohen's $f=.63$; see Table 1 for descriptive statistics), as well as a significant Group $\times$ Time Interaction, $F(1,110)=9.24, p<.004(f=.29)$ indicating that the SOT group became significantly less pessimistic than the control group (see Figure 1) in the 28 days between the Time 1 and Time 2 assessments. The magnitude of this effect corresponds to a medium effect size (Cohen, 1988).

\section{The Moderating Effects of Cognitive Change on Dysphoria}

In this analysis, Time $1 \mathrm{BDI}$ and Time $2 \mathrm{BDI}$ were used as the dependent measures. A significant main effect for Time, $F(1,110)=5.70, p<.03(f=.23)$ was found on depression, corresponding 


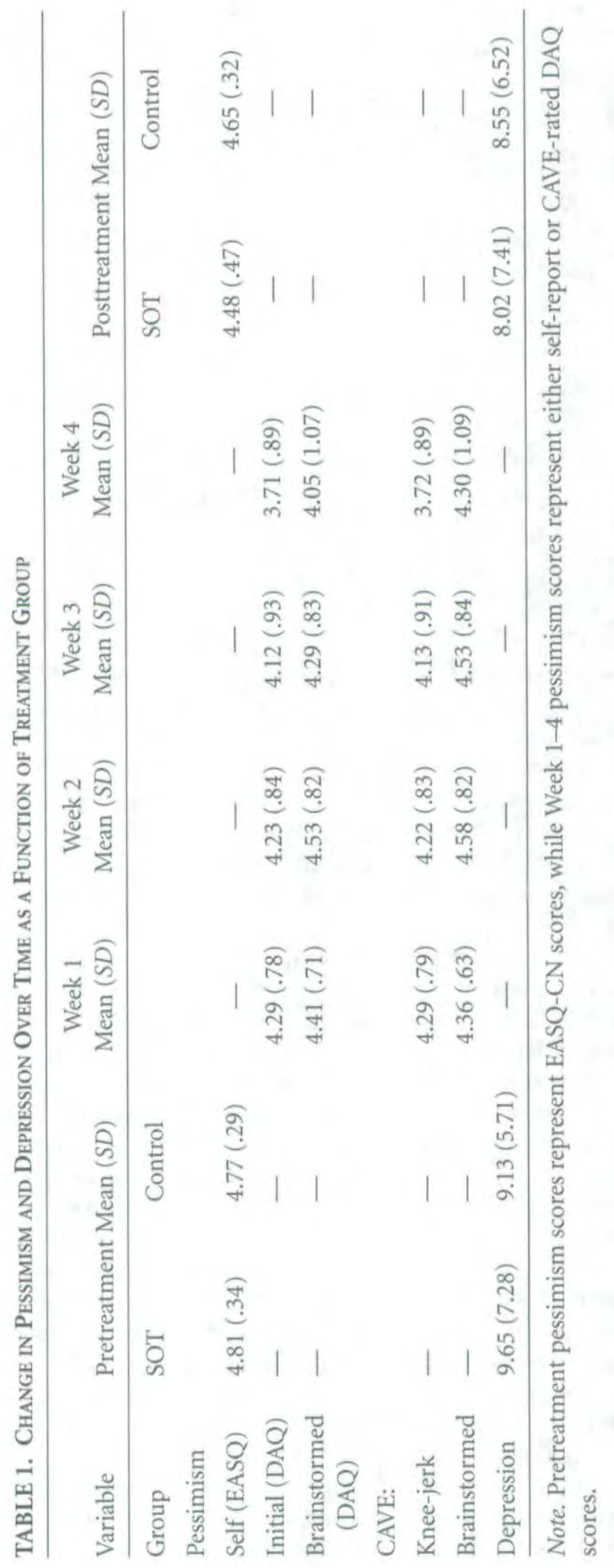




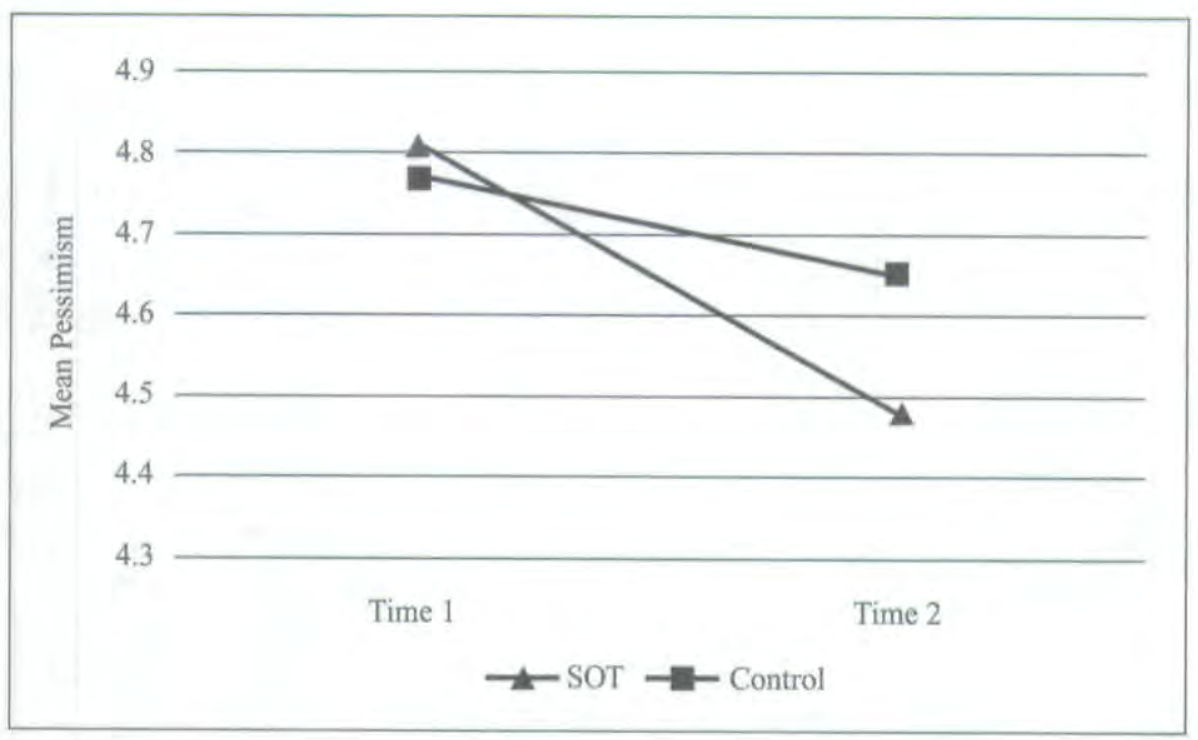

FIGURE 1. Change in pessimism over time as a function of treatment group.

to a medium effect size (see Table 1 for descriptive statistics). The Group $\times$ Time Interaction though, $F(1,110)=1.28$, ns $(f=.11)$, was nonsignificant, indicating that both group experiences drop in depression at the same rate.

To further explore the differential relation of treatment group status and change in depression with explanatory style as a potential moderator, we used an analysis described by Baron and Kenny (1986), but adapted by DeRubeis et al. (1990) specifically for examining explanatory style as a moderator of outcome in cognitive treatments for depression. Hierarchical linear regression was used with the residual change in depression from Time 1 to Time 2 as the dependent variable and residual change in explanatory style and group (SOT $=1$ or control $=0$ ) are predictors entered in step one, with their interaction entered in the second step. This final term represents the differential prediction of change in depression by cognitive change between the two groups, and if significant would indicate the different processes of change that was predicted between the groups. The use of explanatory style as a mediator of change in depressive symptoms was not possible as there was no treatment main effect to mediate (optimism training did not significantly outperform no-treatment in reducing depressive symptoms). In addition, cognitive change and change in depressive symptoms were both made simultaneously (as residual change from Time 1 to Time 2), and so it is impossible to say with certainty which of these two variables "caused" the other. DeRubeis and colleagues (1990), on the other hand, predicted pre- to posttreatment changes in depression from earlier (pre- to midtreatment) cognitive change. As predicted, the interaction term was significant and corresponded to a small to medium effect (see Table 2). Inspection of the correlations between residual change in explanatory style and residual depression change between the treatment and control groups indicates that the relation between these two variables differed between the SOT $(r=0.23)$ and the control group $(r=-0.25$; see Figure 2). The positive correlation between the residual change in explanatory style and change in depression in the SOT group indicated that decreases in pessimistic explanatory style were associated with decreases in symptoms of depression as predicted. Also, the negative correlation between residual change in explanatory style and change in depression found in the control group indicates that as these individuals became less pessimistic, their level of depressive symptoms actually increased. Therefore, it would seem that cognitive change moderated improvement in depressive 
TABle 2. Prediction of Change in Depression From Cognitive Change by TREATMENT GRoup

\begin{tabular}{lrrrcrcc}
\hline Variable & $B$ & SE B & $\beta$ & Cohen's $f^{2}$ & $\Delta R^{2}$ & FChange & $d f$ \\
\hline Step 1 & & & & & .01 & .72 & $(2,109)$ \\
Cognitive change & .77 & 1.31 & .06 & .00 & & & \\
Group & -.79 & .93 & -.08 & .01 & & & \\
Step 2 & & & & & $.05^{*}$ & $5.94^{*}$ & $(1,108)$ \\
Cognitive change $\times$ group & 6.89 & 2.83 & $.43^{*}$ & .06 & & & \\
\hline
\end{tabular}

Note. Cognitive change $=$ Unstandardized residual of explanatory style at Time 2 on explanatory style at Time 1; Group: SOT group $=1$, Control group $=0 ;{ }^{*}=p<.05$; Conventions for $f^{2}:$ small $=.02$, medium $=.15$, large $=.35$.

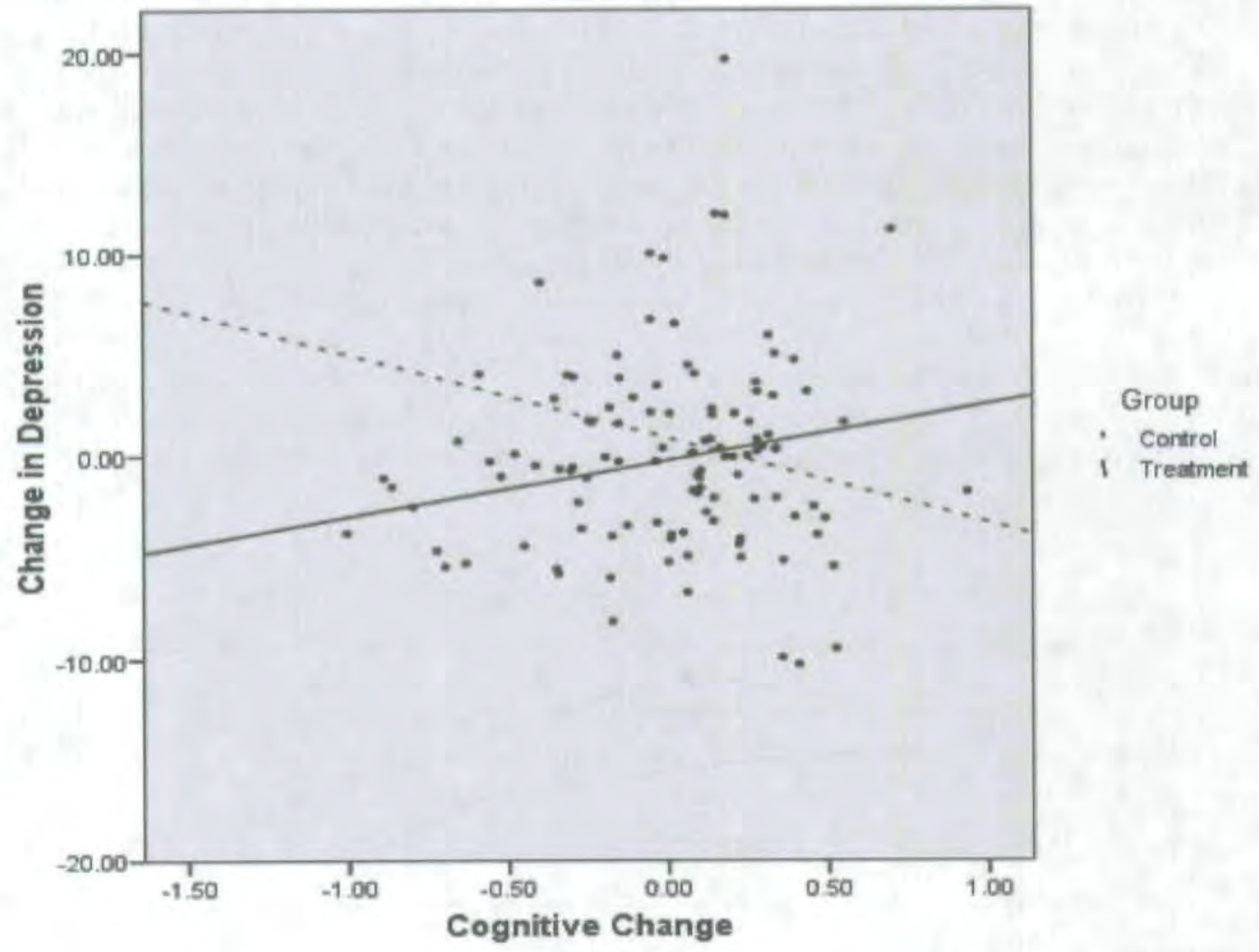

FIGURE 2. Relationship of cognitive change to symptom change as a function of treatment group.

symptoms for the SOT group, as expected. Cognitive moderation, as opposed to cognitive mediation, was tested for insofar as differential reduction in depression scores by cognitive therapy is a necessary prerequisite to establish mediation (DeRubeis et al., 1990), a condition that was not met in the current investigation. One final difference between the present study and DeRubeis et al. (1990) is worthy of note. DeRubeis and colleagues (1990) compared CBT to another active treatment (pharmacotherapy), while we compared SOT with a no-treatment control group. 


\section{Changes in Daily Attributions for SOT Participants}

In addition, the self- and CAVE-rated responses to the 28-day DAQ were subjected to analysis to examine whether attributions for daily, actual events decreased in pessimism over time. Given the number of daily ratings, composite negative scores were computed for each day and then aggregated into weekly averages. In addition, we utilized three within-subject independent variables. The Type variable referred to whether the rating was an initial or a brainstormed rating. The Source variable referred to whether the rating came from the SOT participant (self) or whether it came from a CAVE coder (CAVE). Finally, Time referred to whether a rating was obtained during Week 1, Week 2, Week 3, or Week 4. The Type (2) $\times$ Source $(2) \times$ Time (4) repeated-measures ANOVA revealed a significant main effect for Time on level of pessimistic explanatory style, $F(3,76)=14.62, p<.001(f=.76)$ corresponding to a large effect (see Table 1 for descriptive statistics), while the Time $\times$ Source interaction was nonsignificant, $F(3,76)=0.00, p=n s(f=.00)$. This interaction indicated that although the ratings became less pessimistic with time, this rate of change was equal whether the rating was made by the participant or by an objective other. A Time (4) $\times$ Type (2) repeated-measures ANOVA on level of pessimism, as measured by the DAQ, also found significant main effects for Time, $F(3,50)=3.38, p<.03(f=.45)$, and Type, $F(1,52)=$ $49.02, p<.001$ ( $f=.97$ ), with both of these findings corresponding to large effects (see Table 1 for descriptive statistics), indicating that for the ratings made by the participants themselves, significant drops in pessimism occurred over the 28 days. The Time $\times$ Type interaction, $F(3,50)=3.17$, $p<.04(f=.44)$ was also significant and this result also conformed to a large effect (see Figure 3$)$. Counter to our expectations, pairwise comparisons indicate that the brainstormed ratings were consistently more pessimistic than the initial ratings.

Hierarchical linear modeling (HLM) was utilized in addition to repeated-measures ANOVA to detect the contribution of the different sources of report. The goal of HLM is to predict the rate of change (slope), as well as the pretreatment level of a criterion variable (the intercept), using a prespecified independent variable. The HLM results indicated that a decreasing curvilinear pattern accounted for more variance than a simple, linear pattern and best fit the data for both

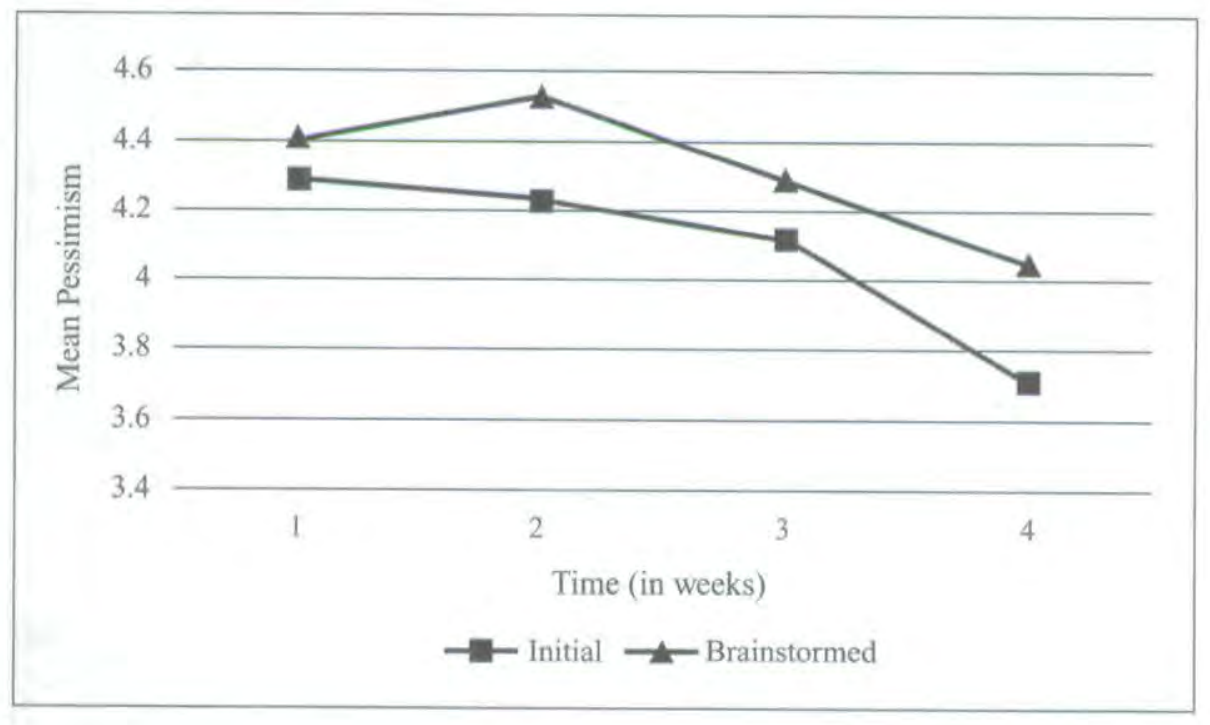

Figure 3. Change in pessimism over time for both initial and brainstormed ratings of causality. 
the initial ( $\beta=-.30$ and .16, respectively, in the Level 1 OLS regression) and brainstormed ratings ( $\beta=-.40$ and .26 , respectively). The HLM results also agreed with the results found using repeated-measures ANOVA and indicated that the source of the rating contributed neither to pretreatment explanatory style [initial rating: $\gamma_{01}=.020, S E=.192, t(49)=.10, p=n s$; brainstormed rating: $\left.\gamma_{01}=.015, S E=.179, t(49)=.08, p=n s\right]$ nor to the rate of change in pessimism over the 28 days [initial rating: $\gamma_{21}=.000, S E=.001, t(2850)=-.31, p=n s$; brainstormed rating: $\left.\gamma_{21}=.000, S E=.001, t(2850)=-.20, p=n s\right]$. Stated more simply, SOT was found to reduce pessimistic explanatory style regardless of the source of report (either the participant or CAVE coder), and pretreatment group differences as a function of source of the report does not appear to explain the increased drop in pessimism for the SOT group. ${ }^{3}$ Additional HLM analyses indicated that attrition from SOT (defined as completing either more than $50 \%$ or $50 \%$ or less of the 28 day DQ ratings) neither contributed to pretreatment explanatory style [initial rating: $\gamma_{01}=-.038$, $S E=.233, t(49)=-.17, p=n s$; brainstormed rating: $\left.\gamma_{01}=-.039, S E=.218, t(49)=-.18, p=n s\right]$ nor to the rate of change in pessimism over the 28 days [initial rating: $\gamma_{21}=.000, S E=.001$, $t(2850)=-.45, p=n s ;$ brainstormed rating: $\left.\gamma_{21}=.000, S E=.001, t(2850)=-.05, p=n s\right]$. Again, stated more simply, SOT attritors were not more pessimistic than SOT completers (consistent with the ANOVA results above) and SOT was found to work equally well if participants completed all, or a subset, of their 28 daily diaries.

\section{DISCUSSION}

The current investigation demonstrated that a minimally supervised, self-directed analogue of self-monitoring and cognitive challenging results in greater drops in pessimism than no treatment. Although SOT as compared to the no-treatment condition did not result in significantly greater decreases in symptoms of depression as compared to levels of depression in control participants, findings indicated that drops in pessimistic explanatory style for SOT participants were associated with the decreases in depression. Further, findings indicated that the greater drops in pessimistic explanatory style found for the SOT group was not due to reporting bias, as equivalent findings were obtained whether self-report or objectively rated materials were used. This finding was obtained with two separate statistical techniques (repeated-measures ANOVA and HLM), lending increased confidence to these results. Equivalent drops in pessimism in SOT were found regardless of whether participants completed all 28 days of the DAQ, or merely a subset of this number. Finally, and counter to expectations, ratings of the causes of events after considerable thought were found to be more pessimistic than ratings done on the spot, despite the fact that both of these ratings became less pessimistic over time for both groups.

Although SOT failed to produce differential decreases in symptoms of depression, this finding was not unexpected given that this was not the primary focus of intervention. Rather, SOT was designed to target a known vulnerability factor for depression, and the findings were consistent with the stated objective. The reformulated learned helplessness theory is a cognitive diathesis-stress model that posits a pessimistic explanatory style, in combination with negative life events, places an individual at greater risk to develop depression. By the end of the 28-day interval during which SOT was administered, the cognitive diathesis component of this model was successfully altered. However, there was no period of time built into the current investigation during which intervening life events could interact with this less pessimistic explanatory style (such as a delayed, post-treatment follow-up assessment). Without both the cognitive diathesis and stress components of the theory, there is no reason to predict significant changes in symptoms of depression. Future SOT research utilizing a delayed follow-up assessment is needed to ensure that reduced risk translates into reductions in symptoms of depression in the long-term. The methodology of the current study was not sufficient to show whether drops in pessimistic explanatory style from participation in SOT resulted in lasting reductions in risk for 
depression. Still, the finding that drops in pessimism were associated with decreases in depression for SOT participants lends credence to our claim that reduced risk should result in statistically significant reductions in depression over a longer interval.

The finding that drops in pessimism were associated with increases in depression for the control group is also worthy of note. Insofar as participants were selected to participate based on their self-reported pessimistic explanatory style, it is possible that the more modest decreases in pessimism observed for the control group were a result of regression to the mean. While the control group experienced drops in both depression and pessimism, the lack of formal intervention for this group might explain why they would continue to develop symptoms of depression. This combination of regression to the mean for pessimism and increasing symptoms of depression would explain why drops in pessimism would be associated with increases in depression for this group. This explanation is purely speculative though, and future research would need to be conducted to both replicate this phenomenon and formally investigate its cause.

However, the use of such a brief, minimally supervised intervention allows the current investigation to address the question of whether psychological intervention can be effective with minimal common or nonspecific mechanisms (Luborsky et al., 1975; Rosenzweig, 1936; Wampold et al., 1997). The SOT is a highly focused intervention, and while participant questions are addressed, the degree of interaction and intimacy necessary for empathy or rapport to be established is sorely lacking. Therefore, the efficacy of SOT is unlikely the result of these mechanisms. Contact with a mental health professional and a common set of goals or methods for treatment are another of these nonspecific mechanisms. While the researchers could be considered a type of mental health professional, contact is, by definition, minimal. Clients are also not instructed on the goals or potential benefits of SOT. However, the methods utilized in SOT are fixed and our participants may have correctly guessed the purpose of the intervention. These facts limit, but certainly do not eliminate, the possibility that client contact and a set of common goals and methods provide the prophylactic benefits of SOT. Future research utilizing computerized administration of SOT will help to completely address this concern. The findings of the current investigation are consistent with prior literature that refutes the idea that all forms of psychotherapy are equally efficacious. Hunsley and DiGiulio (2002) reviewed meta-analytic research traditionally used to support the Dodo Bird's verdict and found extensive evidence for differential efficacy for specific forms of psychotherapy. Other researchers have made similar claims (Cuijpers, van Lier, van Straten, \& Donker, 2005; Stiles, Shapiro, \& Elliott, 1986), and the results for SOT suggest that nonspecific mechanisms may not be the only avenue to the beneficial effects of psychotherapy.

The results of the current investigation indicated that increases in the forethought of causal attributions were associated with the pessimism of these attributions. While this result was unexpected, it is not inconsistent with existing research and theory. It is possible that the process of brainstorming ratings involved perseveratively thinking about problematic situations in a way that was not focused on finding solutions to these problems, but instead served to force the individual to brood on them. Such a ruminative response style, specifically one that more abstractly focuses on problems (Watkins, 2004; Watkins \& Moulds, 2005), has been consistently found to increase depressive affect (Lyubomirsky \& Nolen-Hoeksema, 1995; Lyubomirsky, Tucker, Caldwell, \& Berg, 1999; Morrow \& Nolen-Hoeksema, 1990). These symptoms of depression may, in turn, have primed depressive cognitions (Ingram, 1984; Teasdale, 1988) and resulted in the increases in pessimism obtained. It is interesting to note that while, in this case, rumination produced a negative result in the short-term (increased pessimism), this preceded drops in pessimism that were obtained over successive days. Other researchers have also noted that rumination may not be unambiguously negative, and may lead to more adaptive cognitive processing in the longterm (Watkins, 2004; Watkins \& Moulds, 2005). For instance, it is possible that the short-term ruminative process that we suggest occurred between the initial and brainstormed ratings gave 
way to more active problem solving over the course of the day of the ratings. This process may, in turn, result in the generally more adaptive and less pessimistic response that we observed during the diary entries the following day. Recent research in rumination has attempted to distinguish an active, specific, functional, problem-solving response style from a more passive, self-focused, abstract, and dysfunctional style (Armey et al., in press; Treynor, Gonzalez, \& Nolen-Hoeksema, 2003; Watkins, 2004; Watkins \& Moulds, 2005).

Despite the strengths of the current investigation, certain limitations should be noted. First, there was no delayed, postintervention follow-up assessment to determine whether treatment gains would decay once the intervention was not being actively utilized. It remains a possibility that any decreases in pessimism or depression found quickly vanish after training, limiting the utility and practical efficacy of such a technique. In addition, while we evaluated pretreatment group differences in history of depression, this information was gathered via self-report. As a result, the validity of this information is questionable. Another limitation is that the sample was also composed of relatively high-functioning college students. Although SOT appears efficacious for this population, future research is needed to evaluate whether this intervention is beneficial in a clinical population. This issue is particularly salient given the low intensity and lack of regular therapist intervention in SOT. While these factors are part of what makes SOT so cost-effective and readily disseminated, its efficacy may be significantly diminished with a more symptomatic population. However, it should also be noted that SOT's status as a preventative treatment means that it will be most commonly utilized with a higher functioning, less symptomatic population by definition. Finally, given the disparity in drop-out rates between the SOT and control groups, the possibility exists that this factor influenced the results obtained. Concerns regarding pretreatment group differences resulting from this differential attrition were partially addressed by the finding of pretreatment group equivalence on a large number of variables relevant to treatment. However, the possibility remains that differences could exist on some unmeasured variable(s). The current investigation did not allow for a more detailed investigation of the factors that may have contributed to drop out, however future research involving SOT should examine the reasons why some participants do not complete treatment.

The current investigation is an example of the first stage of behavioral therapy research (Rounsaville et al., 2001) and, as such, is focused primarily on feasibility. Self-administered optimism training has demonstrated the possibility of significant reduction in risk for depression. Insofar as it is an intervention that is inexpensive, can be administered in groups, and does not require extensive training or a health care professional, SOT can assist the large number of individuals who cannot afford and/or do not typically have access to mental health care (New Freedom Commission on Mental Health, 2003; U.S. Department of Health and Human Services, $1999,2002)$. Given the potential SOT has shown here, future research will move forward into stage II, which is focused more on efficacy, with a randomized clinical trial. Future research should also utilize a lengthy follow-up period and take advantage of modern day technology to provide more immediate and ambulatory assessment of daily events. For example, use of the internet as a medium for treatment implementation may hold the potential to reduce the costs associated with SOT and can ensure treatment compliance by automatically recording the time and date that participants complete the DAQ. The use of paper-and-pencil assessment devices completed outside of a laboratory setting provides no safeguards that participants have completed the assessment as instructed (i.e., once a day for 28 days, as opposed to all at once immediately before submitting the questionnaires to the researcher), and can be a limitation of noncomputerized event recording. This increased accountability also has the potential to improve compliance rates. The rate obtained in the current investigation is comparable to that found with other daily diary, cognitively based interventions (Clarke et al., 1992; Helbig \& Fehm, 2004; Primakoff et al., 1986) and elimination of less compliant participants did not alter the results obtained. Both of these observations indicate that noncompliance with the DAQ was not unusual and unlikely to exert a 
large effect on the results obtained. However, replication of the current study and extension using computerized assessment of the DAQ could increase compliance and would increase confidence that SOT is being utilized as described.

In addition to evaluating the efficacy of SOT and utilizing computerized assessment, future research should examine the dose-response curve of SOT. The finding that the rate of decrease in pessimism did not differentiate between participants who completed more than half or less than half of the daily diary entries is troubling and requires further examination. Strong statements about a dose-response relationship for SOT would require either a random sampling of our independent variable of interest (dose) or a purposeful sampling of theoretically meaningful levels. Neither of these two conditions was practical for the current research design given its focus on feasibility. However, future research could administer SOT of varying durations to randomly assigned participants. Such as study could more definitively determine if a relationship exists between treatment duration and outcome as well as the ideal duration of treatment.

Finally, the current investigation focused on the reformulated learned helplessness concept of attributional style. However, other similar cognitive theories of the etiology of depression that currently boast a similar amount of empirical support (Abramson et al., 1999; Alloy et al., 1999; Alloy \& Clements, 1998; Alloy, Just, \& Panzarella, 1997; Hankin, Abramson, Miller, \& Haeffel, 2004; Reilly-Harrington, Alloy, Fresco, \& Whitehouse, 1999). Hopelessness theory (Abramson et al., 1989) posits that individuals who, when exposed to negative life events, evidence a negative inferential style are at greater risk to develop depression. Inferential style, like attributional style, is defined as attributing negative events to stable and global causes. However, unlike attributional style, the event does not have to be attributed to an internal cause, the individual has to infer that negative consequences will result from the negative life event, and to infer that the event implies that one is fundamentally flawed or worthless. Future research is necessary to determine if SOT results in decreased risk for depression, as defined by both the reformulated learned helplessness and hopelessness theories.

\section{NOTES}

1. These results are available from the corresponding author upon request.

2. Results were also obtained without the imputation of missing data and imputing data only for those participants with $50 \%$ or more data on the DAQ. Under both of these conditions, essentially identical findings were obtained. These results are available from the first author upon request.

3. Unfortunately, differences in rate of change could not be investigated between initial and brainstormed ratings in HLM insofar as each individual contributed ratings to each level, and the groups are therefore not independent. The HLM, similar to ANOVA, assumes that levels of all independent variables are independent of one another.

\section{REFERENCES}

Abela, J. R. Z., Fresco, D. M., Kostelnik, B. A., \& Payne, A. V. L. (2003). Assessment of attributional style: Further comparisons of the ASQ and CAVE technique. Manuscript under review.

Abela, J. R. Z., \& Seligman, M. E. P. (2000). The hopelessness theory of depression: A test of the diathesisstress component in the interpersonal and achievement domains. Cognitive Therapy and Research, 24, 361-378.

Abramson, L. Y., Alloy, L. B., Hogan, M.E., Whitehouse, W. G., Donovan, P., Rose, D., et al. (1999). Cognitive vulnerability to depression: Theory and evidence. Journal of Cognitive Psychotherapy: An International Quarterly, 13, 5-20.

Abramson, L. Y., Metalsky, G. I., \& Alloy, L. B. (1989). Hopelessness depression: A theory-based subtype of depression. Psychological Review, 96, 358-372. 
Abramson, L. Y., Seligman, M. E. P., \& Teasdale, J. D. (1978). Learned helplessness in humans: Critique and reformulation. Journal of Abnormal Psychology, 87, 49-74.

Alloy, L. B., \& Abramson, L. Y. (1988). Depressive realism: Four theoretical perspectives. In L. B. Alloy (Ed.), Cognitive processes in depression. New York: Guilford.

Alloy, L. B., Abramson, L. Y., Whitehouse, W. G., Hogan, M. E., Tashman, N., Steinberg, D., et al. (1999). Depressogenic cognitive styles: Predictive validity, information processing and personality characteristics, and developmental origins. Behavior Research and Therapy, 37, 503-531.

Alloy, L. B., \& Clements, C. M. (1998). The hopelessness theory of depression: Tests of the symptom component. Cognitive Therapy and Research, 22, 303-335.

Alloy, L. B., Just, N., \& Panzarella, C. (1997). Attributional style, daily life events, and hopelessness depression: Subtype validation by prospective variability and specificity of symptoms. Cognitive Therapy and Research, 21, 321-344.

American Psychiatric Association. (2000). Diagnostic and statistical manual of mental disorders (4th ed., text revision). Washington, DC: American Psychiatric Association.

Armey, M. F., Moore, M. T., Fresco, D. M., Mennin, D. S., Turk, C. L., Heimberg, R. G., et al. (in press). Brooding and pondering: Isolating the active ingredients of depressive rumination with exploratory factor analysis and structural equation modeling. Assessment.

Baron, R. M., \& Kenny, D. A. (1986). The moderator-mediator variable distinction in social psychological research: Conceptual, strategic, and statistical considerations. Journal of Personality and Social Psychology, 51, 1173-1182.

Beck, A. T. (1967). Depression: Clinical, experimental, and theoretical aspects. New York: Harper \& Row.

Beck, A. T. (1987). Cognitive models of depression. Journal of Cognitive Psychotherapy: An International Quarterly, 1, 5-37.

Beck, A. T., Rush, A. J., Shaw, B. F., \& Emery, G. (1979). Cognitive therapy of depression. New York: Guilford.

Beck, A. T., Steer, R. A., \& Garbin, M. (1988). Psychometric properties of the Beck depression inventory: Twenty-five years of evaluation. Clinical Psychology Review, 8, 77-100.

Blackburn, I., \& Moorhead, S. (2001). Depression. In W. L. Lyddon \& J. V. Jones (Eds.), Empirically supported cognitive therapies: Current and future applications. New York: Springer Publishing.

Clarke, G. N., Hops, H., Lewinsohn, P. M., Andrews, J., Seeley, J. R., \& Williams, J. (1992). Cognitivebehavioral group treatment of adolescent depression: Prediction of outcome. Behavior Therapy, 23, 341-354.

Cohen, J. (1988). Statistical power analysis for the behavioral sciences (2nd ed.). Hillsdale, NJ: Erlbaum.

Colin, S., Sweeney, P. D., \& Schaeffer, D. E. (1981). The causality of causal attributions in depression: A crosslagged panel correlational analysis. Journal of Abnormal Psychology, 90, 14-22.

Cuijpers, P., van Lier, P. A. C., van Straten, A., \& Donker, M. (2005). Examining differential effects of psychological treatment of depressive disorder: An application of trajectory analysis. Journal of Affective Disorders, 89, 137-146.

DeRubeis, R. J., \& Crits-Cristoph, P. (1998). Empirically supported individual and group psychological treatments for adult mental disorders. Journal of Consulting and Clinical Psychology, 66, 37-52.

DeRubeis, R. J., Evans, M. D., Hollon, S. D., Garvey, M. J., Grove, W. M., \& Tuason, V. B. (1990). How does cognitive therapy work? Cognitive change and symptom change in cognitive therapy and pharmacotherapy for depression. Journal of Consulting and Clinical Psychology, 58, 862-869.

Dobson, K. S., Hollon, S. D., Dimidjian, S., Schmaling, K. B., Kohlenberg, R. J., Gallop, R. J., et al. (2008). Randomized trial of behavioral activation, cognitive therapy, and antidepressant medication in the prevention of relapse and recurrence in major depression. Journal of Consulting and Clinical Psychology, 76, 468-477.

Fresco, D. M., \& Craighead, L. W. (1993). Self-administered optimism training. Unpublished manual. Chapel Hill: University of North Carolina.

Hankin, B. L., Abramson, L. Y., Miller, N., \& Haeffel, G. J. (2004). Cognitive vulnerability-stress theories of depression: Examining affective specificity in the prediction of depression versus anxiety in three prospective studies. Cognitive Therapy and Research, 28, 309-345. 
Helbig, S., \& Fehm, L. (2004). Problems with homework in CBT: Rare exception or rather frequent? Behavioural and Cognitive Psychotherapy, 32, 291-301.

Hovarth, A. O., \& Greenberg, L. S. (1994). The working alliance: Theory, research, and practice. New York: Wiley.

Hunsley, J., \& DiGiulio, G. (2002). Dodo bird, phoenix, or urban legend? The question of psychotherapy equivalence. The Scientific Review of Mental Health Practice, 1, 11-22.

Ingram, R. E. (1984). Toward an information-processing analysis of depression. Cognitive Therapy and Research, 8, 442-477.

Lafferty, P., Beutler, L. E., \& Crago, M. (1991). Differences between more and less effective psychotherapists: A study of select therapist variables. Journal of Consulting and Clinical Psychology, 57, 76-80.

Liang, J., \& Bentler, P. M. (2004). An EM algorithm for fitting two-level structural equation models. Psychometrika, 69, 101-122.

Luborsky, L., Singer, B., \& Luborsky, L. (1975). Comparative studies of psychotherapies. Is it true that "everyone has won and all must have prizes"? Archives of General Psychiatry, 32, 995-1008.

Lyubomirsky, S., \& Nolen-Hoeksema, S. (1995). Effects of self-focused rumination on negative thinking and interpersonal problem solving. Journal of Personality and Social Psychology, 69, 176-190.

Lyubomirsky, S., Tucker, K. L., Caldwell, N. D., \& Berg, K. (1999). Why ruminators are poor problem solvers: Clues from the phenomenology of dysphoric rumination. Journal of Personality and Social Psychology, 77, 1041-1060.

Metalsky, G. I., Joiner, T. E., Hardin, T. S., \& Abramson, L. Y. (1993). Depressive reactions to failure in a naturalistic setting: A test of the hopelessness and self-esteem theories of depression. Journal of Abnormal Psychology, 102, 101-109.

Moore, M. T., \& Fresco, D. M. (2007). Depressive realism and attributional style: Implications for individuals at risk for depression. Behavior Therapy, 38, 144-154.

Morrow, J., \& Nolen-Hoeksema, S. (1990). Effects of responses to depression on the remediation of depressive affect. Journal of Personality and Social Psychology, 58, 519-527.

New Freedom Commission on Mental Health. (2003). Achieving the promise: Transforming mental health care in America. Final report. DHHS Pub. No. SMA-03-3832. Rockville, MD.

Oei, T. P. S., \& Kazmierczak, T. (1997). Factors associated with dropout in a group cognitive behavioral therapy for mood disorders. Behaviour Research and Therapy, 35, 1025-1030.

Peterson, C. (1991). The meaning and measurement of explanatory style. Psychological Inquiry, 2, 1-10.

Peterson, C., Bettes, B. A., \& Seligman, M. E. P. (1985). Depressive symptoms and unprompted causal attributions: Content analysis. Behaviour Research and Therapy, 23, 379-382.

Peterson, C., Schulman, P., Castellon, C., \& Seligman, M. E. P. (1992). The explanatory style scoring manual. In C. P. Smith (Ed.), Handbook of thematic content analysis (pp. 383-392). New York: Cambridge.

Peterson, C., \& Seligman, M. E. P. (1984). Causal explanations as a risk factor for depression: Theory and evidence. Psychological Review, 91, 347-374.

Peterson, C., Semmel, A., von Baeyer, C., Abramson, L. Y., Metalsky, G. I, \& Seligman, M. E. P. (1982). The attributional style questionnaire. Cognitive Therapy and Research, 6, 287-299.

Peterson, C., \& Villanova, P. (1988). An expanded attributional style questionnaire. Journal of Abnormal Psychology, 97, 87-89.

Primakoff, L., Epstein, N., \& Covi, L. (1986). Homework compliance: An uncontrolled variable in cognitive therapy outcome research. Behavior Therapy, 17, 433-446.

Reilly-Harrington, N. A., Alloy, L. B., Fresco, D. M., \& Whitehouse, W. G. (1999). Cognitive styles and life events interact to predict bipolar and unipolar symptomatology. Journal of Abnormal Psychology, 108, $567-578$.

Riskind, J. H., Castellon, C. S., \& Beck, A. T. (1989). Spontaneous causal explanations in unipolar depression and generalized anxiety: Content analysis of dysfunctional-thought diaries. Cognitive Therapy and Research, 13, 97-108. 
Robins, C. J. (1988). Attributions and depression: Why is the literature so inconsistent? Journal of Personality and Social Psychology, 54, 880-889.

Rosenzweig, S. (1936). Some implicit common factors in diverse methods in psychotherapy. Journal of Orthopsychiatry, 6, 412-415.

Rounsaville, B. J., Carroll, K. M., \& Onken, L. S. (2001). A stage model of behavioral therapies research: Getting started and moving on from stage I. Clinical Psychology: Science and Practice, 8, 133-142.

Schulman, P., Castellon, C., \& Seligman, M. E. P. (1989). Assessing explanatory style: The content analysis of verbatim explanations and the attributional style questionnaire. Behaviour Research and Therapy, $27,505-512$.

Seligman, M. E. P., Abramson, L. Y., Semmel, A., \& von Baeyer, C. (1979). Depressive attributional style. Journal of Abnormal Psychology, 88, 242-247.

Simons, A. D., Levine, J. L., Lustman, P. J., \& Murphy, G. E. (1984). Patient attrition in a comparative outcome study of depression. Journal of Affective Disorders, 6, 163-173.

Smith, M. L., Glass, G. V., \& Miller, T. I. (1980). The benefits of psychotherapy. Baltimore: Johns Hopkins University Press.

Snyder, C. R., Michael, S. T., \& Cheavens, J. (1999). Hope as a psychotherapeutic foundation of common factors, placebos, and expectancies. In M. A. Huble, B. Duncan, \& S. Miller (Eds.), Heart and soul of change (pp. 179-200). Washington, DC: American Psychological Association.

Stiles, W. B., Shapiro, D. A., \& Elliott, R. (1986). Are all psychotherapies equivalent? American Psychologist, $41,165-180$.

Stukenberg, K. W., Dora, J. R., \& Kiecolt-Glaser, J. K. (1990). Depression screening scale validation in an elderly, community-dwelling population. Psychological Assessment, 2, 134-138.

Sweeney, P. D., Anderson, K., \& Bailey, S. (1986). Attributional style in depression: A meta-analytic review. Journal of Personality and Social Psychology, 50, 974-991.

Teasdale, J. D. (1988). Cognitive vulnerability to persistent depression. Cognition and Emotion, 2, 247-274.

Treynor, W. F. C., Gonzalez, R., \& Nolen-Hoeksema, S. (2003). Rumination reconsidered: A psychometric analysis. Cognitive Therapy and Research, 27, 247-259.

U.S. Department of Health and Human Services. (1999). Mental health: A report of the surgeon generalexecutive summary. Rockville, MD: U.S. Department of Health and Human Services, Substance Abuse and Mental Health Services Administration, Center for Mental Health Services, National Institutes of Health, National Institute of Mental Health.

U.S. Department of Health and Human Services. (2002). One department serving rural America: Rural task force report to the secretary. Retrieved March 11,2009, from http://www.ruralhealth.hrsa.gov/PublicReport.htm

Wampold, B. E., Mondin, G. W., Moody, M., Stich, F., Benson, K., \& Ahn, H. (1997). A meta-analysis of outcome studies comparing bona fide psychotherapies: Empirically, "All must have prizes." Psychological Bulletin, 122, 203-215.

Watkins, E. (2004, November). Training mode of thinking to manipulate rumination. Paper presented at the annual meeting of the Association for Advancement of Behavior Therapy, New Orleans, LA.

Watkins, E., \& Moulds, M. (2005). Distinct modes of ruminative self-focus: Impact of abstract versus concrete rumination on problem solving in depression. Emotion, 5, 319-328.

Acknowledgments. Portions of this article were presented at the annual meeting of the Society for Research in Psychopathology in Toronto, Ontario, Canada, and the Association for Behavioral and Cognitive Therapies in Washington, DC.

Correspondence regarding this article should be directed to David M. Fresco, Department of Psychology, Kent State University, Kent, OH 44240.E-mail:fresco@kent.edu 
Copyright of Journal of Cognitive Psychotherapy is the property of Springer Publishing Company, Inc. and its content may not be copied or emailed to multiple sites or posted to a listserv without the copyright holder's express written permission. However, users may print, download, or email articles for individual use. 